\title{
Exact Analysis of Dispersive SAW Devices on ZnO/Diamond/Si-Layered Structures
}

\author{
Tsung-Tsong Wu and Yung-Yu Chen
}

\begin{abstract}
In this paper, a formulation for calculating the effective permittivity of a piezoelectric layered SAW structure is given, and the exact frequency response of $\mathrm{ZnO} /$ diamond/Si-layered $\mathrm{SAW}$ is calculated. The effective permittivity and phase velocity dispersion of a $\mathrm{ZnO} /$ diamond/Si -layered half space are calculated and discussed. The frequency response of an unapodized SAW transducer is calculated, and the center frequency shift caused by the velocity dispersion is explained. In addition, the electromechanical coupling coefficients of the $\mathrm{ZnO} /$ diamond/Si -layered half space based on two different formulas are calculated and discussed. Finally, based on the results of the study, we propose an exact analysis for modeling the layered SAW device. The advantage of using the effective permittivity method is that, not only the null frequency bandwidth, but also the center frequency shift and insertion loss can be evaluated.
\end{abstract}

\section{INTRODUCTION}

$\mathrm{P}$ ROPAGATION of surface waves in layered structures has been of interest in the development of dispersive SAW devices. By including a high velocity diamond layer between a piezoelectric layer and a Si substrate, the surface wave velocity can be increased significantly [1], [2]. This results in an increase in the SAW frequency without a decrease in the electrode spacing into the submicron region. In addition, layered SAW also preserves the advantages of high coupling coefficient and tiny temperature coefficient [3].

In the last decade, there are experimental as well as theoretical investigations on the layered SAW. In the theoretical calculation, Adler [4], [5] presented a matrix approach for studying SAW and pseudo SAW in layered piezoelectric media. Later, based on the matrix method, Adler and Solie [6] calculated the electromechanical coupling of layered SAW with $\mathrm{ZnO}$ on diamond. In the calculations, different boundary conditions were considered. By using the conventional approach for calculating surface wave dispersion, Nakahata et al. [7] reported analyses of phase velocity and electromechanical coupling coefficient for three different layered SAWs with diamond as the middle layer. In a subsequent paper, Nakahata et al. [8] also reported experimental results on a $2.5-\mathrm{GHz}$ SAW filter on diamond.

\footnotetext{
Manuscript received September 26, 2000; accepted July 20, 2001. Financial support of this research was provided by the National Science Council of ROC through the grant NSC89-2218-E-002-033.

The authors are with the Institute of Applied Mechanics, National Taiwan University, Taipei, Taiwan (e-mail: wutt@spring.iam.ntu.edu.tw)
}

As to the modeling of the SAW transducer, Hachigo and Malocha [9] used the delta function model to calculate the null frequency bandwidth of $\mathrm{ZnO} /$ diamond/Si-layered SAW and found the reduction of the bandwidth caused by velocity dispersion. In addition, they presented the dispersive equivalent circuit model for layered structures.

In this paper, the frequency response of a $\mathrm{ZnO} /$ diamond/ Si-layered SAW was calculated using the effective permittivity approach. The formulation based on the matrix method [10]-[13] for calculating the effective permittivity of a layered piezoelectric medium is given. The effective permittivity and phase velocity dispersion of a $\mathrm{ZnO} /$ diamond/Si-layered half space was calculated. The frequency response of an unapodized SAW transducer was then calculated based on the effective permittivity method, and discussions were given on the shifting of the center frequency. The electromechanical coupling coefficients of the $\mathrm{ZnO} /$ diamond/Si-layered half space based on two different formulas are compared and discussed. Finally, based on the results of the study, we propose an exact analysis for modeling the layered SAW device.

\section{Effective Permittivity of A LAYered Piezoelectric Medium}

In the quasi-static approximation, the governing field equations of piezoelectricity can be expressed as

$$
\begin{aligned}
\sigma_{i j, j} & =\rho \ddot{u}_{i} \\
D_{i, i} & =0
\end{aligned}
$$

where $\rho$ is the mass density and $D_{i}$ is the electric displacement. $\sigma_{i j}$ and $u_{i}$ are the Cauchy stress tensor and the mechanical displacement, respectively. The piezoelectric constitutive equations with the displacement $u_{i}$ and the electric potential $\phi$ as variables are of the form

$$
\begin{aligned}
\sigma_{i j} & =C_{i j k l} u_{k, l}+e_{l i j} \phi_{, l} \\
D_{i} & =e_{i k l} u_{k, l}-\varepsilon_{i l} \phi_{, l} .
\end{aligned}
$$

In these equations, $C_{i j k l}$ is the elastic stiffness tensor at constant electric field of the piezoelectric medium, $e_{l i j}$ is the piezoelectric tensor, and $\varepsilon_{i l}$ is the dielectric permittivity tensor at constant strain. For plane harmonic waves propagating in the $\mathrm{x}$-direction of a planar layered system (Fig. 1), the mechanical displacement vector $\mathbf{u}$, the z-component of the electric displacement vector $\mathbf{D}_{\mathbf{z}}$, 


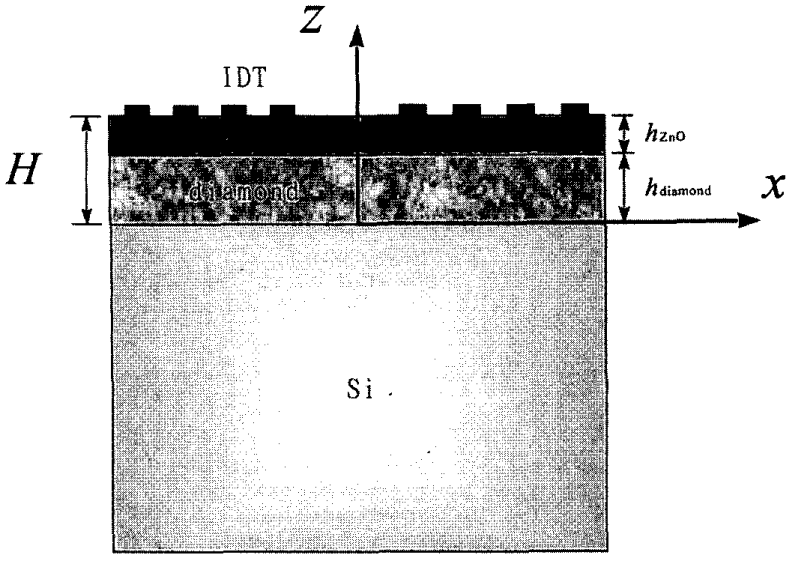

Fig. 1. Coordinates of the planar-layered half space.

the traction vector $\mathbf{t}$, and the electric potential $\phi$ can be expressed in the forms:

$$
\begin{aligned}
\mathbf{u}(x, z, t) & =\overline{\mathbf{u}}(z) \exp \left\{i\left(\omega t-k_{x} x\right)\right\} \\
\mathbf{D}_{\mathbf{z}}(x, z, t) & =\bar{D}_{z}(z) \exp \left\{i\left(\omega t-k_{x} x\right)\right\} \\
\mathbf{t}(x, z, t) & =\overline{\mathbf{t}}(z) \exp \left\{i\left(\omega t-k_{x} x\right)\right\}, \text { and } \\
\phi(x, z, t) & =\bar{\phi}(z) \exp \left\{i\left(\omega t-k_{x} x\right)\right\}, \text { respectively, }
\end{aligned}
$$

where $\omega$ is the circular frequency and $k_{x}$ is the wave number along the x-direction of the plane harmonic wave. $\overline{\mathbf{u}}(z)$, $\bar{D}_{\mathbf{z}}, \overline{\mathbf{t}}$, and $\bar{\phi}$ are the amplitudes of $\mathbf{u}, \mathbf{D}_{\mathbf{z}}, \mathbf{t}$, and $\phi$, respectively. According to the state vector formulation of elastic waves in anisotropic solids, the governing equations $[(1)-$ (4)] can be arranged in the matrix form as [10]-[13]:

$$
\frac{d}{d z} \bar{\xi}(z)=-i \mathbf{N}(z) \cdot \bar{\xi}(z), \quad \text { with } \bar{\xi}(z)=\left\{\begin{array}{c}
\overline{\mathbf{u}}(z) \\
\bar{\phi}(z) \\
i \overline{\mathbf{t}}(z) \\
i \bar{D}(z)
\end{array}\right\}
$$

where $\mathbf{N}$ is called the fundamental acoustic tensor. The solution of (9) can be expressed in terms of the propagator matrix (or called transfer matrix), $e^{-i \mathbf{N} z}$, and the initial condition at $\mathrm{z}=0, \bar{\xi}(0)$, as

$$
\bar{\xi}(z)=e^{-i \mathbf{N} z} \cdot \bar{\xi}(0) .
$$

This solution is appropriate for a multilayered system, because the overall solution can be obtained by multiplication of the solutions for each layer. However, numerical difficulties are encountered for large frequency-thickness values, for both growing and decaying potentials are mixed in the propagator matrix. For multilayered media, the surface impedance approach can be adopted to avoid the numerical difficulties [12], [13]. Using this approach, the generalized traction vector $\mathbf{T}=\left(i \overline{\mathrm{t}}, i \bar{D}_{z}\right)^{T}$ and the generalized displacement vector $\mathbf{U}=(\overline{\mathbf{u}}, \bar{\phi})^{T}$ are defined at a plane normal to the z-axis. On using the eigenvalue-eigenvector

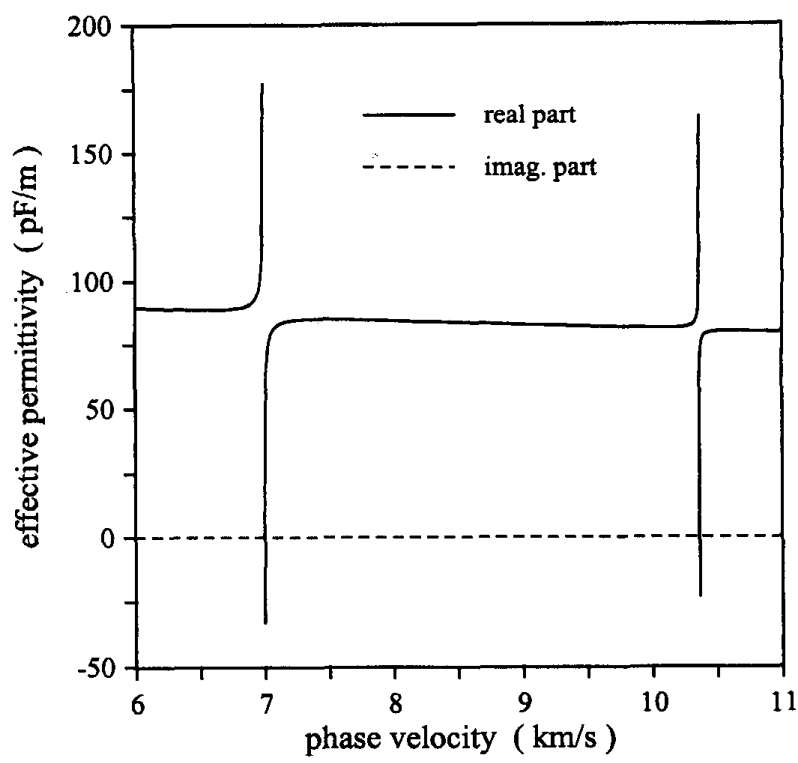

Fig. 2. Plot of the effective permittivity of a $\mathrm{ZnO} /$ diamond/Silayered half space as a function of the phase velocity. $f=1000 \mathrm{MHz}$, $h_{\text {diamond }}=22 \mu \mathrm{m}$, and $h_{Z n O}=0.9 \mu \mathrm{m}$.

decomposition of the transfer matrix, (10) can be arranged in the form

$$
\left\{\begin{array}{l}
\mathbf{U} \\
\mathbf{T}
\end{array}\right\}=\left[\begin{array}{ll}
\mathbf{A}_{1} & \mathbf{A}_{2} \\
\mathbf{L}_{1} & \mathbf{L}_{2}
\end{array}\right]\left[\begin{array}{cc}
\Phi_{1} & \mathbf{0} \\
\mathbf{0} & \Phi_{2}
\end{array}\right]\left\{\begin{array}{l}
\mathbf{C}_{1} \\
\mathbf{C}_{2}
\end{array}\right\}
$$

where $\mathbf{A}_{1}, \mathbf{A}_{2}, \mathbf{L}_{1}, \mathbf{L}_{2}$ are $4 \times 4$ matrices, and $\left[\begin{array}{ll}\mathbf{A}_{1} & \mathbf{A}_{2} \\ \mathbf{L}_{1} & \mathbf{L}_{2}\end{array}\right]$ is the matrix of eigenvectors of $\mathbf{N}$, which are arranged such that the first four eigenvalues of $\mathbf{N}$ correspond to waves decaying in the positive $\mathbf{z}$-direction. $\mathbf{C}_{1}, \mathbf{C}_{2}$ are constant vectors and are related to the initial conditions at $\mathrm{z}=0$. The diagonal matrices are

$$
\begin{aligned}
& \Phi_{1}(z)=\operatorname{diag}\left(e^{-i k_{z 1} z}, e^{-i k_{z 2} z}, e^{-i k_{z 3} z}, e^{-i k_{z 4} z}\right) \\
& \Phi_{2}(z)=\operatorname{diag}\left(e^{-i k_{z 5} z}, e^{-i k_{z 6} z}, e^{-i k_{z 7} z}, e^{-i k_{z 8} z}\right)
\end{aligned}
$$

with $k_{z i}$ the eigenvalues of $\mathbf{N}$. With the above arrangements of the transfer matrix, the surface impedance tensor of the layered piezoelectric medium can be defined as [13]

$$
\mathbf{T}=i \omega \mathbf{G} \mathbf{U}
$$

where the surface impedance tensor $\mathbf{G}$ is a $4 \times 4$ matrix, which can be derived from the eigenvalue-eigenvector decomposition. The recursive formulas of $\mathbf{G}$ for layered media are shown in Appendix A for convenience.

For convenience in defining the effective permittivity, the surface impedance tensor $\mathbf{G}$ can be arranged in the form

$$
\mathbf{G}=\left[\begin{array}{cc}
\mathbf{G}_{m} & \mathbf{g}_{T \phi} \\
\mathbf{g}_{D U}^{T} & \mathbf{g}_{D \phi}
\end{array}\right]
$$




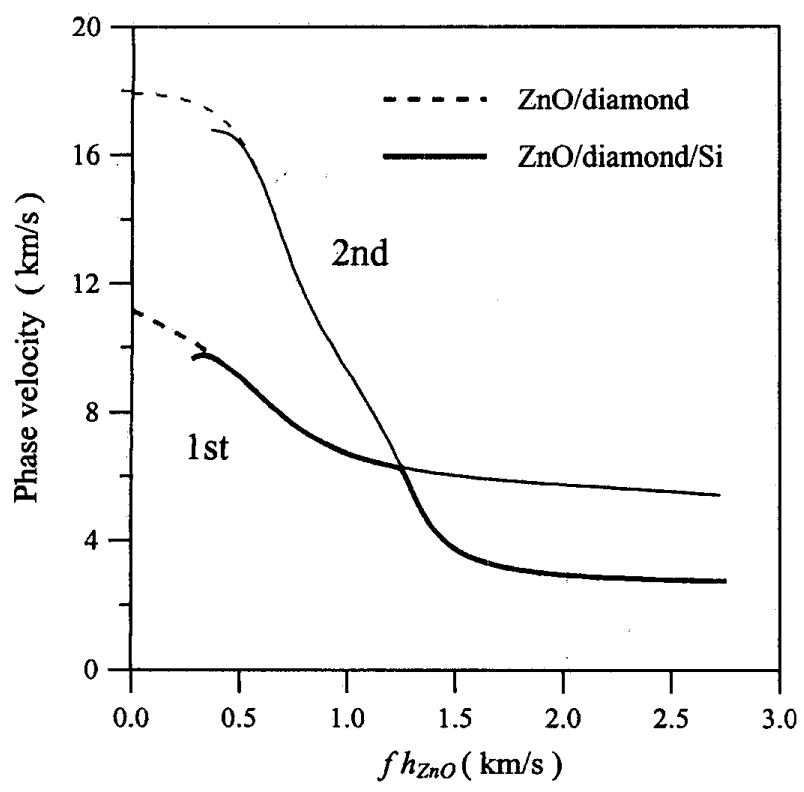

Fig. 3. Phase velocity dispersion of the first two SAW modes with free surface boundary condition. $h_{\text {diamond }}=22 \mu \mathrm{m} ; h_{Z_{n O}}=0.9 \mu \mathrm{m}$.

where $\mathbf{G}_{m}$ is the mechanical part of $\mathbf{G}$ and is a $3 \times 3$ matrix. $\mathbf{g}_{T \phi}$ and $\mathbf{g}_{D U}^{T}$ are $3 \times 1$ vectors. $\mathbf{g}_{T \phi}$ is the electromechanical coupling between the traction and the electric potential, and $\mathbf{g}_{D U}^{T}$ relates the electric displacement to the particle displacement. The superscript " $\mathrm{T}$ " in $\mathbf{g}_{D U}^{T}$ denotes the transpose. The scalar $\mathbf{g}_{D \phi}$ relates the electric potential to the electric displacement. Eq. (14) can then be expanded as

$$
\begin{aligned}
i \overline{\mathbf{t}} & =i \omega\left[\mathbf{G}_{m} \overline{\mathbf{u}}+\mathbf{g}_{T \phi} \bar{\phi}\right] \\
i \bar{D}_{z} & =i \omega\left[\mathbf{g}_{D U}^{T} \overline{\mathbf{u}}+\mathbf{g}_{D \phi} \bar{\phi}\right] .
\end{aligned}
$$

Consider a $\mathrm{ZnO} /$ diamond/Si-layered half space in contact with a vacuum, the traction on the top surface of $\mathrm{ZnO}$ must be zero. The electric displacement at $z=$ $h_{\text {diamond }}+h_{Z n O}=H$ in the solid side is denoted as $\left.\bar{D}_{Z}\right|_{Z=H^{-}}$(Fig. 1) and is given by

$$
\left.\bar{D}_{Z}\right|_{Z=H^{-}}=\left.\omega\left(-\mathbf{g}_{D U}^{T} \mathbf{G}_{m}^{-1} \mathbf{g}_{T \phi}+\mathbf{g}_{D \phi}\right) \bar{\phi}\right|_{Z=H^{-}} .
$$

In the vacuum, $z>H$, the electric potential satisfies the Laplace equation. The normal electric displacement $\left.\bar{D}_{Z}\right|_{Z=H^{+}}$at $z=h$ in the vacuum side is given by $\left.\bar{D}_{Z}\right|_{Z=H^{+}}=\left.\varepsilon_{0} k_{x} \bar{\phi}\right|_{Z=H^{\prime}}$. The effective permittivity at the interface between the vacuum and $\mathrm{ZnO}$ can then be written as

$\varepsilon_{s}=\frac{\left.\bar{D}_{Z}\right|_{Z=H^{+}}-\left.\bar{D}_{Z}\right|_{Z=H^{-}}}{\left.k_{x} \bar{\phi}\right|_{Z=H}}=\varepsilon_{0}-\frac{\left.\bar{D}_{Z}\right|_{Z=H^{-}}}{\left.k_{x} \bar{\phi}\right|_{Z=H}}$,

where the continuity of the electric potential at the interface has been employed.
Shown in Fig. 2 is a plot of the effective permittivity of a $\mathrm{ZnO} /$ diamond/Si-layered half space. In the calculation, the frequency was chosen as $1000 \mathrm{MHz}$, and the thickness of the diamond layer was $22 \mu \mathrm{m}$ and; of the $\mathrm{ZnO}$ layer was $0.9 \mu \mathrm{m}$. In the figure, the solid line represents the real part of effective permittivity, and the broken line represents the imaginary part. The effective permittivity is real in this case, which indicates that there is no acoustic energy leaked into the substrate. The plot shows that for phase velocities around $7000 \mathrm{~m} / \mathrm{s}$ (the first generalized SAW mode solution), a pole and a zero are found. Similar pole and zero are found for phase velocity close to $10000 \mathrm{~m} / \mathrm{s}$ (the second SAW mode). The zeros correspond to the surface wave solution for a free surface, because the charge density is zero. The poles indicate the surface wave solution for a metallized surface, because it gives a finite charge density at zero electric potential.

Shown in Fig. 3 is the phase velocity dispersion of the first two SAW modes (first mode: thicker solid line; second mode: thinner'solid line) of the $\mathrm{ZnO} /$ diamond/Si-layered half space with free surface boundary condition. The dispersion was calculated based on the aforementioned effective permittivity approach. In Fig. 3, the thicker dashed line represents the phase velocity dispersion of the first mode of a $\mathrm{ZnO} /$ diamond-layered half space, and the thinner dashed line is the second mode. The results show that the diamond layer can be treated as a half space so far as $f h_{Z n O}$ value is high enough. (In this case, for the first mode, the separation $f h_{Z n O}$ value is around $350 \mathrm{~m} / \mathrm{s}$.) It is worth noting that the dispersion curves of the first two SAW modes come very close to each other but do not cross as mentioned in [6]. At frequency-thickness $f h_{Z n O}=1247 \mathrm{~m} / \mathrm{s}(1386 \mathrm{MHz})$, there exists high curvature bend for both of the first two modes. Instead of decreasing smoothly, the first mode of surface acoustic wave bend down at $f h_{Z n O}=1247 \mathrm{~m} / \mathrm{s}$, and the second mode bend up. As will be shown later, the frequency at which the phase velocity changes sharply corresponds to a sharp change in the electromechanical coupling coefficient for both modes.

\section{FREQUenCy Responses of A ZnO/Diamond/Si-SAW DEvicE}

The frequency response of an unapodized transducer using the effective permittivity method can be expressed as [14]

$$
H_{t}(\omega)=\left(\omega W \Gamma_{s}\right)^{\frac{1}{2}} \overline{\rho_{e}}(\beta) \exp \left(-\frac{1}{2} j \beta L\right)
$$

where $W$ is the overlap length of the IDT electrodes and L is the length of the unapodized transducer. The wavenumber $\beta(\omega)$ is taken to be $\frac{\omega}{v_{0}(\omega)}$, where $v_{0}$ is the free surface velocity (open circuit velocity). It is worth noting that for this layered system, $v_{0}$ is a function of frequency $\omega$ and can 


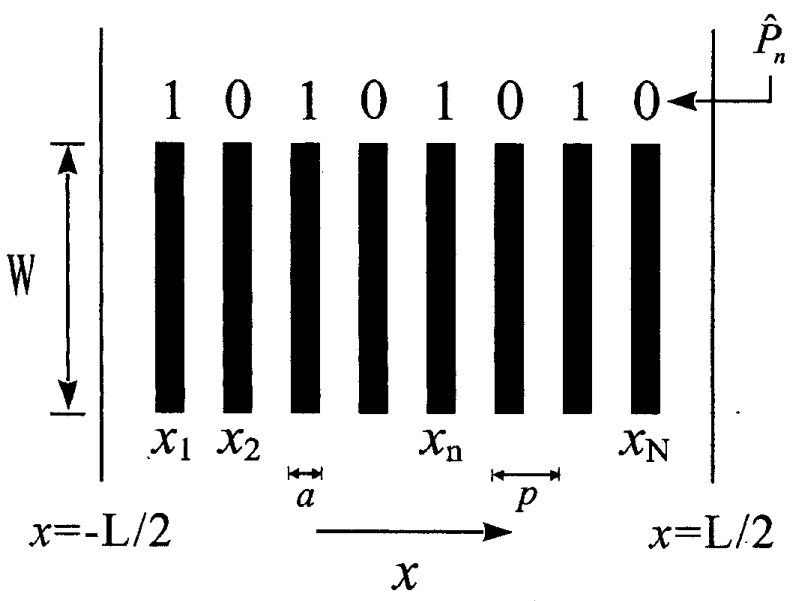

Fig. 4. The polarity arrangement of the IDT transducer.

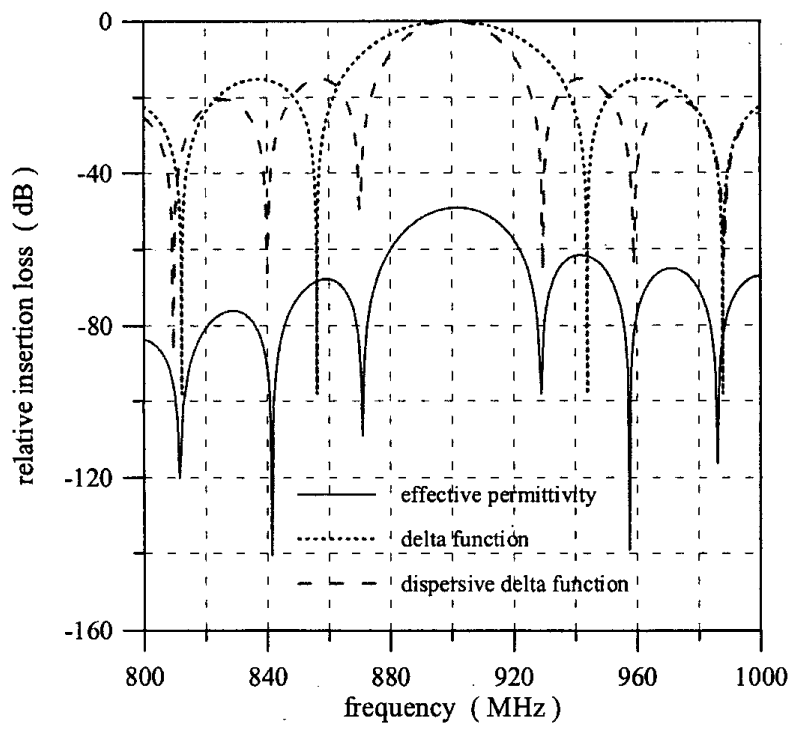

Fig. 5. Frequency responses of an unapodized transducer (20 pairs) on a $\mathrm{ZnO} /$ diamond/Si-layered structure calculated by delta function, dispersive delta function [9], and the effective permittivity approach.

be obtained from the dispersion relation. The function $\Gamma_{s}$ is defined as $[14]$

$$
\frac{1}{\Gamma_{s}}=-\beta\left[\frac{d \varepsilon_{s}\left(k_{x}\right)}{d k_{x}}\right]_{\beta}
$$

where $k_{x}$ is the wave number along the $\mathrm{x}$-direction of the plane harmonic wave defined in Section II.

The Fourier transform of the electrostatic charge density $\bar{\rho}_{e}(\beta)$ for IDT transducers with regular electrodes can be expressed as $[14$, eq. (4.85)]

$$
\bar{\rho}_{e}(\beta)=\bar{A}_{f}(\beta) \bar{\rho}_{f}(\beta)
$$

where $\bar{A}_{f}(\beta)$ and $\bar{\rho}_{f}(\beta)$ are the Fourier transform of the array factor and the elemental charge density, respectively.

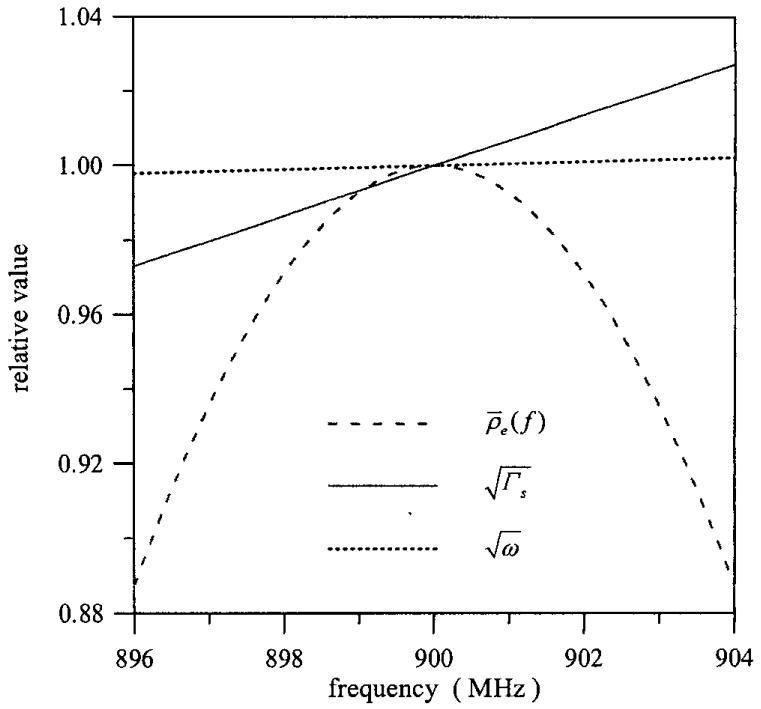

Fig. 6. Frequency dependence of $\sqrt{\Gamma_{s}}, \sqrt{\omega}$, and $\bar{\rho}_{e}\left(k_{0}\right)$ in the neighborhood of the center frequency.

The array factor is represented by

$$
\bar{A}_{f}(\beta)=\sum_{n=1}^{N} \hat{P}_{n} \exp \left(-i \beta x_{n}\right)
$$

where $\hat{P}_{n}$ is defined in Fig. 4 . The elemental charge density of an IDT with regular electrodes is listed in Appendix B for convenience.

Fig. 5 shows the frequency responses of an unapodized transducer on a $\mathrm{ZnO} /$ diamond/Si-layered structure based on the delta function (dotted line), dispersive delta function (dashed line) [9], and the effective permittivity (solid line) models. The thickness of the diamond layer is $22 \mu \mathrm{m}$, and that of the $\mathrm{ZnO}$ layer is $0.9 \mu \mathrm{m}$. The center frequency was chosen at $900 \mathrm{MHz}$, and the number of electrode pairs was 20 . The results showed that both the dispersive delta function model and the effective permittivity model could show the reduction of the null frequency bandwidth. A close examine of the frequency response calculated by the effective permittivity model reveals that there is a center frequency shift from 900 to $901.92 \mathrm{MHz}$. The reason for this shifting of the center frequency may be explained from (20), in which there are three frequency-dependent functions $\sqrt{T_{s}}, \sqrt{\omega}$, and $\bar{\rho}_{e}(\beta)$. Fig. 6 shows the calculated frequency dependence of the functions $\sqrt{\Gamma_{s}}, \sqrt{\omega}$, and $\bar{\rho}_{e}(\beta)$ in the neighborhood of the center frequency. Results showed that $\sqrt{\omega}$ is a slow-varying function in this frequency region, and $\bar{\rho}_{e}(\beta)$ is symmetrical with respect to the center frequency. The function $\sqrt{\Gamma_{s}}$ is asymmetrical with respect to the center frequency and monotonically increases. Therefore, we note that the slight increase of the center frequency is mainly due to the monotonical increase of $\sqrt{\Gamma_{s}}$ on the frequency for this case. In the last section of this paper, we will propose a way to avoid this kind of center frequency shifting. 


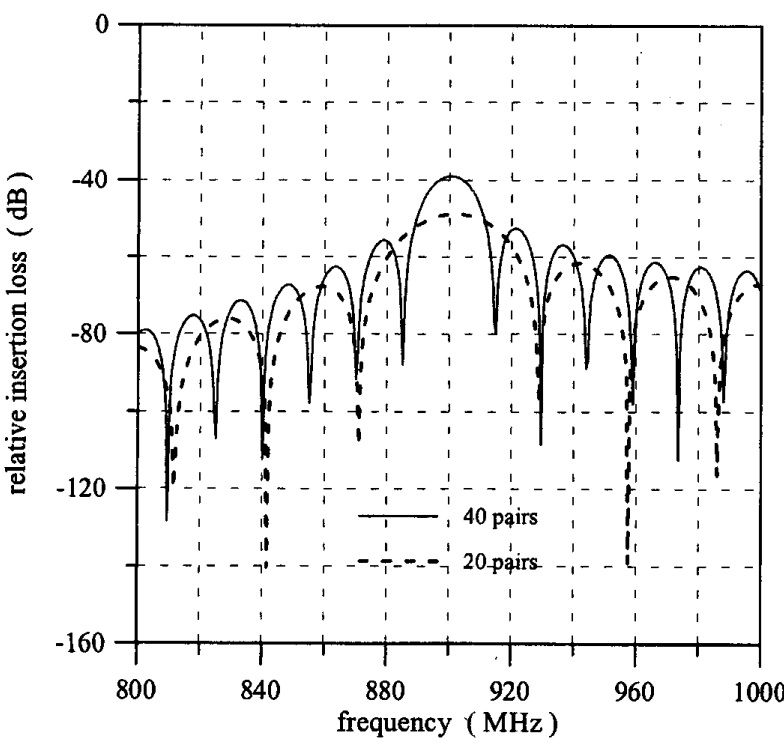

Fig. 7. Frequency responses of unapodized IDT transducers with 20 (broken line) and 40 (solid line) electrode pairs.

Fig. 7 shows the calculated frequency responses of unapodized IDT transducers with 20 (broken line) and 40 (solid line) electrode pairs. The results show that the center frequency of the 40 pairs IDT is $900.48 \mathrm{MHz}$, and that of the 20 pairs is $901.92 \mathrm{MHz}$. Therefore, it is obvious that the shift of the center frequency of an IDT transducer can be minimized by increasing the number of IDT pairs. In addition, we note that by using the effective permittivity approach, the insertion loss can be obtained as $-48.96 \mathrm{~dB}$ (20 pairs) and $-38.92 \mathrm{~dB}$ (40 pairs).

\section{The Electromechanical Coupling CoEfFicient}

The electromechanical coupling coefficient $K_{s}^{2}$ can be defined as [15]

$$
K_{s}^{2}=2 \Gamma_{s} \varepsilon_{s}^{(\infty)}
$$

where $\varepsilon_{s}^{(\infty)}$ is the effective permittivity at infinite slowness and $\Gamma_{s}$ can be calculated exactly from (24). By using the Ingebrigtsen approximation [16], the electromechanical coupling coefficient $K_{s}^{2}$ of a piezoelectric medium can be approximated as

$$
K_{s}^{2}=2 \frac{v_{0}-v_{m}}{v_{0}}
$$

where $v_{m}$ is the surface wave velocity with metallized surface and $v_{0}$ is the free surface wave velocity defined as before.

The solid lines shown in Fig. 8 are portions of the dispersion curves of the $\mathrm{ZnO} /$ diamond/Si-layered half space mentioned in Section II. The thicker solid line is the first,

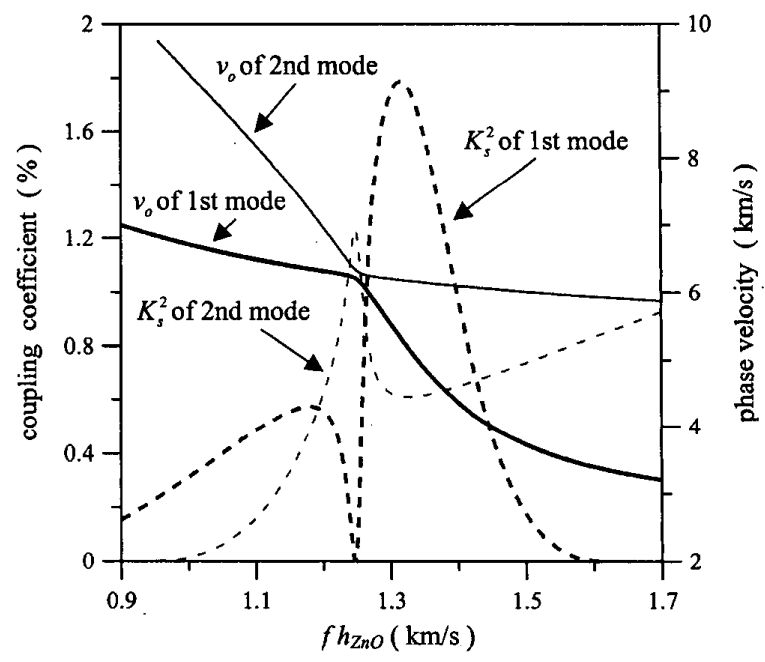

Fig. 8. Electromechanical coupling coefficients (dashed lines) and dispersion curves of the first two SAW mode (solid lines) of the $\mathrm{ZnO} /$ diamond/Si-layered half space.

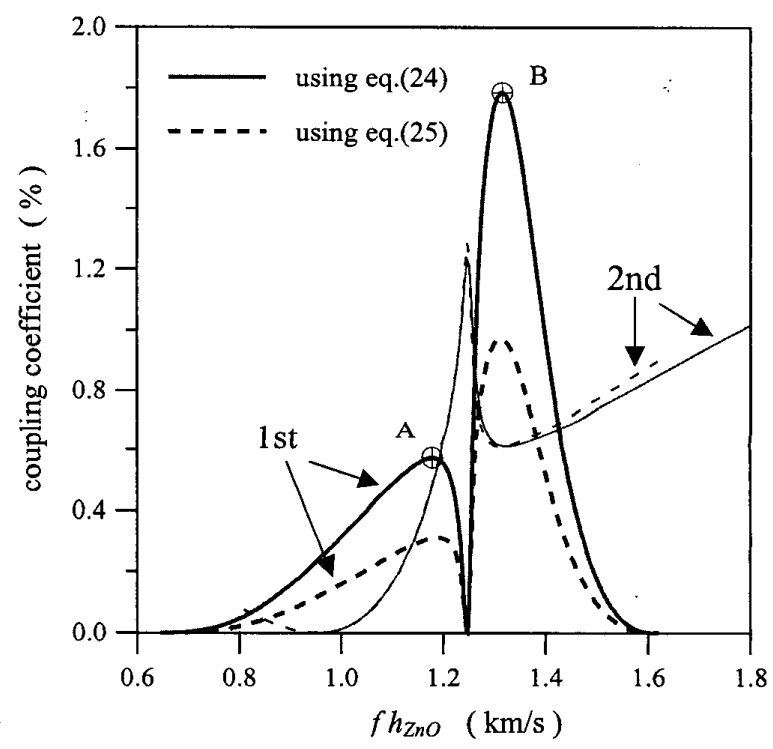

Fig. 9. Coupling coefficients of the $\mathrm{ZnO} /$ diamond/Si-layered structure calculated by using (24) (solid lines) and (25) (dashed lines).

and the thinner solid line is the second SAW mode. Based on (24), the electromechanical coupling coefficients for the two first SAW modes are calculated: the thicker dashed line represents that of the first SAW mode, and the thinner dashed line represents the second SAW mode. The results show, for both modes, at $f h_{Z n O}=1247 \mathrm{~m} / \mathrm{s}(1386 \mathrm{MHz})$, where the phase velocity changes sharply, in accordance with the frequency, where the electromechanical coupling coefficient changes dramatically. It is worth noting that at this sharp change frequency, $K_{s}^{2}$ of the first mode goes to almost zero, and that of the second mode goes to a maximum. 


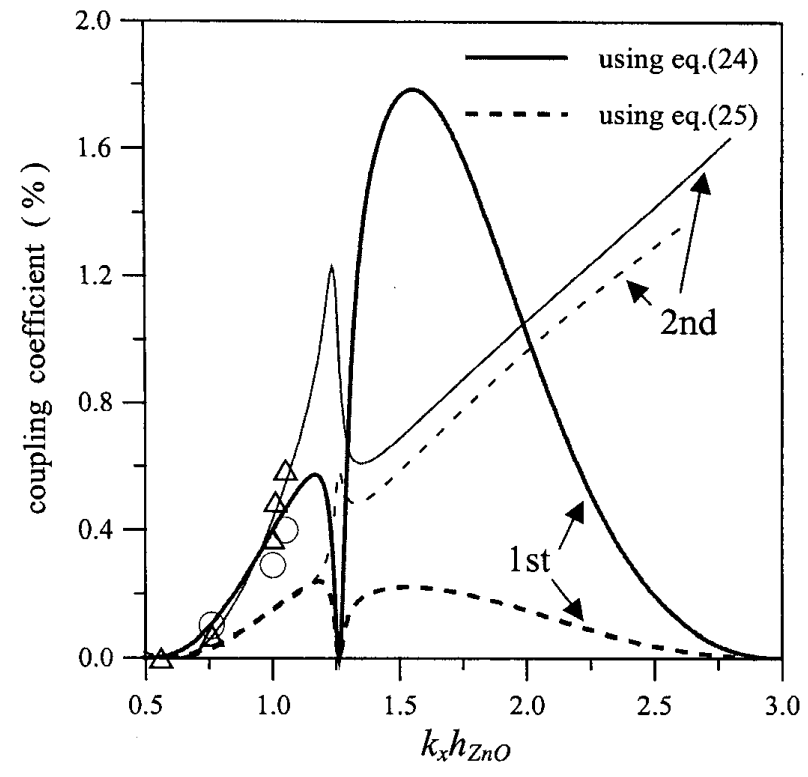

Fig. 10. Coupling coefficient of the $\mathrm{ZnO} /$ diamond/Si-layered structure calculated at constant wavelength.

Study on the electromechanical coupling coefficient for SAWs with $\mathrm{ZnO}$ on diamond-layered structure has been made by Adler and Solie [6]. They pointed out that the coupling coefficient calculated at constant frequency is about four times larger than that calculated at constant wavelength. They also suggested that, for a dispersive structure, the independent variable is frequency, and the constant frequency value should be used.

We note that the calculation of the electromechanical coupling coefficient $K_{s}^{2}$ reported in [6] is based on (25). In the following, we will show that if $K_{s}^{2}$ is calculated using (24), the difference caused by calculation at constant frequency and constant wavelength disappear. Fig. 9 shows the coupling coefficients of the $\mathrm{ZnO} /$ diamond/Si-layered structure calculated by using (25) (dashed line) and (24) (solid line) at constant frequency. The coupling coefficients of the first mode are denoted by thicker lines, and those of the second mode are denoted by thinner lines. The IDT transducer is assumed to place on top of the $\mathrm{ZnO}$ layer (Fig. 1). The results showed that the coupling coefficient of the first mode (thicker line) calculated based on (25) is much smaller than that based on (24). For the second mode, the difference between these two ways of calculation is smaller than that of the first mode. Fig. 10 shows coupling of the same structure calculated at constant wavelength. In this situation, it is clearly seen that, for the first mode, the difference of coupling calculated based on (24) and (25) is even bigger than that of at constant frequency. The open circles and triangles in Fig. 10 are the experimental $K_{s}^{2}$ of the first and second SAW modes reported by Nakahata et al. [7]. Results showed that the experimental values are well in accordance with those calculated from (24).

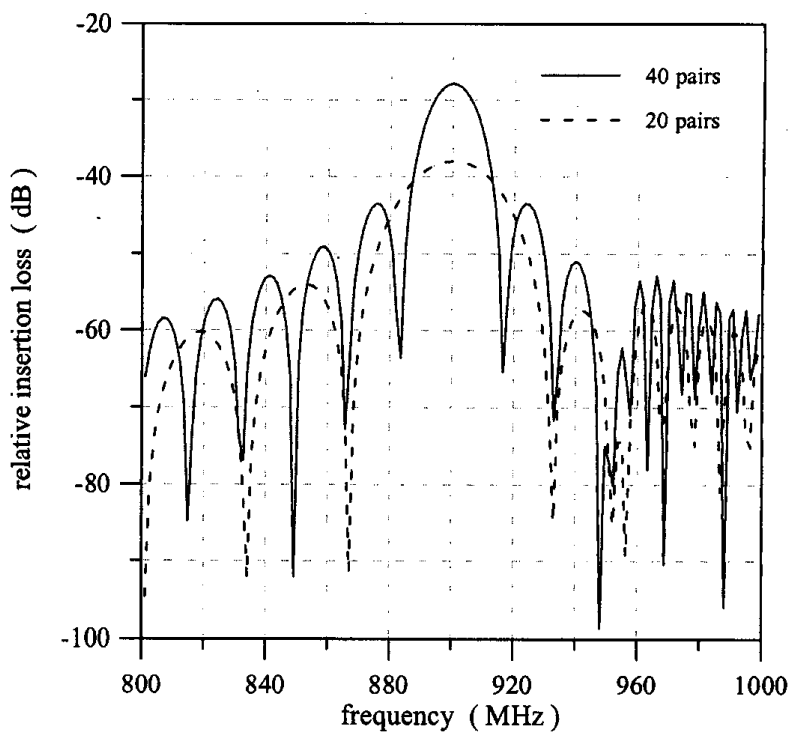

Fig. 11. Frequency response of $\mathrm{ZnO} /$ diamond/Si-layered $\mathrm{SAW}$ with 20 and 40 electrode pairs. $\mathrm{f}=900 \mathrm{MHz}, h_{\text {diamond }}=22 \mu \mathrm{m}$, and $h_{Z n O}=1.31 \mu \mathrm{m}$

Comparisons made between Fig. 9 and 10 show that the coupling coefficients of both modes calculated at constant frequency and at constant wavelength are exactly the same so far as (24) is adopted. We note that the main difference of (24) and (25) is that, in (25), $\Gamma_{s}$ is approximated by Ingebrigtsen's approximation, and in (24), $\Gamma_{s}$ is calculated exactly.

\section{Preliminary Design of a Dispersive SAW}

For the IDT transducer described in Section III, the thickness of the $\mathrm{ZnO}$ layer was $0.9 \mu \mathrm{m}$, and the design center frequency was $900 \mathrm{MHz}$. Previous discussions showed that, with this configuration, there is a shift of the center frequency from 900 to $901.92 \mathrm{MHz}$. In the neighborhood of the center frequency, the function $\sqrt{\Gamma_{s}}$ is monotonically increasing and was suspected to be the reason for the center frequency shift.

To avoid the shifting of the center frequency, the operating frequency of an IDT SAW transducer should be chosen at one of the two local maxima (points A or B) of the coupling coefficient plot shown in Fig. 9. For the values of the electromechanical coupling coefficient $K_{s}^{2}$ around points $\mathrm{A}$ and $\mathrm{B}$ are symmetrical with respect to the frequencies of points A and B. Therefore, from (24), the values of the function $\sqrt{\Gamma_{s}}$ are also symmetrical. It is worth noting that, from Fig. 8, the velocity dispersion around the first $K_{s}^{2}$ maximum (point A in Fig. 9) is less dispersive when compared with that of the second maximum (point B in Fig. 9). So, it is better to choose point A in practice. For example, on choosing the thickness frequency $f h_{Z n O}=1179 \mathrm{~m} / \mathrm{s}$ (point A in Fig. 9), the thickness of the $\mathrm{ZnO}$ layer is 
$1.31 \mu \mathrm{m}$ for a $900-\mathrm{MHz}$ IDT transducer. Fig. 11 shows the frequency response of such a layered SAW with 20 and 40 electrode pairs. Results demonstrated that the center frequency is not shifted and is equal to $900 \mathrm{MHz}$ as designed. Another advantage of choosing $f h_{Z h O}$ value at point $\mathrm{A}$ of the coupling coefficient curve is that the insertion loss can be reduced significantly as shown in Fig. 11.

\section{ConClusion}

An analysis of the $\mathrm{ZnO} /$ diamond/Si-layered SAW based on the effective permittivity approach is presented. The formulation based on the matrix method for calculating the effective permittivity of a layered piezoelectric medium is given and used to calculate the phase velocity dispersion and the electromechanical coupling coefficient of the layered system. In the layered SAW simulation, in addition to the bandwidth narrowing reported in [9], we found that there exists a central frequency shift caused by the phase velocity dispersion. The frequency shift is mainly due to the asymmetry of the function $\sqrt{\Gamma_{s}}$ around the operating frequency. On the other hand, in the calculation of the electromechanical coefficient, results show that the sharp change of phase velocity in the dispersion curve is intimately related to the sharp change of the electromechanical coupling coefficient. We have shown that if the electromechanical coupling coefficient is calculated based on the exact effective permittivity function (24) directly, the coupling coefficients calculated at constant frequency and constant wavelengths are equivalent. Finally, we proposed a method, based on the effective permittivity approach, to design a dispersive IDT SAW transducer with zero center frequency shift and low insertion loss.

\section{APPENDIX A}

The surface impedance of the $j$ th layer is [13]

$$
\begin{gathered}
\mathbf{G}_{j}(z)=\left[\mathbf{Z}_{1 j} \mathbf{W}_{1 j}(z) \mathbf{R}_{j-1} \mathbf{W}_{2 j}^{-1}(z)+\mathbf{Z}_{2 j}\right] \\
{\left[\mathbf{W}_{1 j}(z) \mathbf{R}_{j-1} \mathbf{W}_{2 j}^{-1}(z)+\mathbf{I}\right]^{-1}} \\
\mathbf{Z}_{\alpha j}=-\frac{1}{\omega} \mathbf{L}_{\alpha j} \mathbf{A}_{\alpha j}^{-1} \\
\mathbf{W}_{\alpha j}(z)=\mathbf{A}_{\alpha j} \Phi_{\alpha j}(z) \mathbf{A}_{\alpha j}^{-1}, \alpha=1,2 \\
\mathbf{R}_{j-1}=\left[\mathbf{Z}_{1 j}-\mathbf{G}_{j-1}\right]^{-1}\left[\mathbf{G}_{j-1}-\mathbf{Z}_{2 j}\right]
\end{gathered}
$$

where $Z_{a j}$ are local impedances for the up-going wave and the down-going wave. $\alpha=1$ represents decaying waves in the positive $\mathrm{z}$-direction (up-going), and $\alpha=2$ represents decaying waves in the negative z-direction (down-going).

\section{Appendix B}

Consider an infinite array of regular electrodes with width $a$ and pitch $p$, the elemental charge density function is $[14]$

$$
\begin{gathered}
\overline{\rho_{f}}(\beta)=\left(\varepsilon_{0}+\varepsilon_{p}\right) \frac{2 \sin (\pi s)}{P_{-s}(-\cos \Delta)} P_{n}(\cos \Delta) \\
\text { for } n \leq \frac{\beta p}{2 \pi} \leq n+1
\end{gathered}
$$

where $\Delta=\frac{\pi a}{p}$, and $s=\frac{\beta p}{2 \pi}-n, 0 \leq s \leq 1 . \varepsilon_{p}=$ $\left(\varepsilon_{11} \varepsilon_{33}-\varepsilon_{13}^{2}\right)^{\frac{1}{2}}$ where $\varepsilon_{i j}$ is the permittivity tensor of the top piezoelectric layer.

\section{REFERENCES}

[1] K. Yamanouchi, N. Sakuri, and T. Satoh, "SAW propagation characteristics and fabrication technology of piezoelectric thin film/diamond structure," in Proc. IEEE Ultrason. Symp., pp. 351-354, 1989

[2] H. Nakahata, K. Higaki, A. Hachigo, S. Shikata, N. Fukmori, Y. Takahashi, T. Kajihara, and Y. Yamamoto, "High frequency surface acoustic wave filter using $\mathrm{ZnO} /$ diamond/Si-structure," Jpn. J. Appl. Phys., Part 1, vol. 33, no. 1, pp. 324-328, 1994.

[3] H. Nakahata, K. Higaki, A. Hachigo, S. Fujii, T. Uemura, and S. Shikata, "Diamond SAW filter for 2.488 Gbps retiming," in Proc. IEEE Ultrason. Symp., pp. 319-322, 1998.

[4] E. L. Adler, "Matrix methods applied to acoustic waves in multilayers," IEEE Trans. Ultrason., Ferroelect., Freq. Contr., vol. 37 , no. 6 , pp. $485-490,1990$

[5] - "SAW and pseudo-SAW properties using matrix methods," IEEE Trans. Ultrason., Ferroelect., Freq. Contr., vol. 41, no. 5 , pp. 699-705, 1994.

[6] E. L. Adler and L. Solie, "ZnO on diamond: SAWs and pseudoSAWs," in Proc. IEEE Ultrason. Symp., pp. 341-344, 1995.

[7] H. Nakahata, A. Hachigo, K. Higaki, S. Fujii, S. Shikata, and N. Fujimori, "Theoretical study on SAW characteristics of layered structures including a diamond layer," IEEE Trans. Ultrason. Ferroelect., Freq. Contr., vol. 42, no. 3, pp. 362-375, 1995.

[8] H. Nakahata, K. Higaki, S. Fujii, A. Hachigo, H. Kitabayashi, K. Tanabe, Y. Seki, and S. Shikata, "SAW devices on diamond," in Proc. IEEE Ultrason. Symp., pp. 361-370, 1995.

[9] A. Hachigo and D. C. Malocha, "SAW device modeling including velocity dispersion based on $\mathrm{ZnO} /$ diamond/Si-layered structures," IEEE Trans. Ultrason., Ferroelect., Freq. Contr., vol. 45, no. 3, pp. $660-665,1998$.

[10] A. N. Stroh, "Steady state problems in anisotropic elasticity," J. Math. Phys., vol. 41, pp. 77-103, 1962.

[11] A. H. Fahmy and E. L. Adler, "Propagation of acoustic surface waves in multilayers: A matrix description," Appl. Phys. Lett., vol. 22 , no. 10 , pp. $495-497$, May 1973.

[12] K. A. Ingebrigtsen and A. Tonning, "Elastic surface waves in crystals," Phys. Rev., vol. 184, no. 3, pp. 942-951, 1969.

[13] B. Honein, A. M. B. Braga, P. Barbone, and G. Hermann, "Wave propagation in piezoelectric layered media with some applications," J. Intelligent Mater. Syst. Struct., vol. 2, no. 4, pp. $542-557,1991$.

[14] D. P. Morgan, Surface-Wave Devices for Signal Processing. New York: Elsevier, 1991.

[15] H. Matthews, Surface Wave Filters: Design, Construction, and Use. New York: Wiley, p. 66, 1977.

[16] K. A. Ingebrigtsen, "Surface waves in piezoelectrics," J. Appl. Phys., vol. 40, pp. 2681-2686, 1969. 


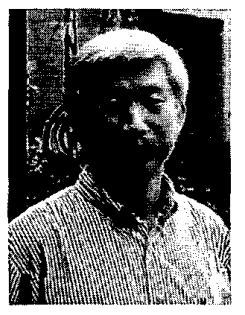

Tsung-Tsong Wu received his B.S. degree from National Taiwan University in 1977 and M.S. and Ph.D. degrees in theoretical and applied mechanics from Cornell University in 1983 and 1987, respectively. He joined the faculty of National Taiwan University in 1987 and currently is professor of the Institute of Applied Mechanics.

His research interests are in ultrasonic wave propagation and related precision measurement techniques. Particular interests in-

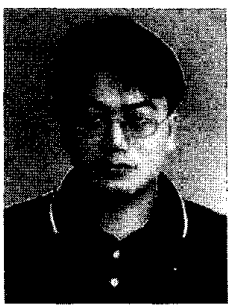

Yung-Yu Chen received his B.S. degree from National Chen-Kung University in 1977 and currently is Ph.D. candidate of the Institute of Applied Mechanics, National Taiwan University.

His research interests are surface waves in layered anisotropic and/or piezoelectric materials, SAW devices, and related sensors.

and/or piezoelectric laser ultrasonics, and nondestructive evaluation of materials.

$\mathrm{He}$ is a member of American Society of Mechanical Engineering and American Society for Nondestructive Testing. 